\title{
AS CARACTERÍSTICAS DA DOR EM PORTADORES DE INSUFICIÊNCIA RENAL CRÔNICA EM PROGRAMA DE HEMODIÁLISE
}

\author{
Lúcio Silva ${ }^{1}$ \\ Alexandre Tourino Mendonça ${ }^{2}$ \\ Lilian de Almeida Carvalho ${ }^{3}$
}

\begin{abstract}
1 Médico Nefrologista, Mestre em Ciências da Saúde.
2 Doutor em Ciências dos Alimentos.

3 Nutricionista Clínica, Mestre em Ciências da Saúde.
\end{abstract}

\section{Recebido em: 20/05/2013 - Aprovado em: 30/07/2013 - Disponibilizado em: 15/08/2013}

\section{RESUMO}

Este artigo descreve uma pesquisa sobre a dor em pacientes com insuficiência renal crônica em programa de hemodiálise. Foram selecionados 40 pacientes adultos, com diagnóstico de insuficiência renal crônica estágio V. A seleção de pacientes foi feita através de dados de prontuário e exames laboratoriais. Foi utilizado um desenho experimental observacional, realizado em 20 pacientes em programa de hemodiálise ambulatorial na Clínica CenemgeBelo Horizonte/MG, e em 20 pacientes portadores de insuficiência renal crônica internados no Hospital Belo HorizonteBelo Horizonte/MG. As análises descritivas consideraram os pacientes inicialmente em um único grupo, "de portadores de insuficiência renal crônica, em hemodiálise". Em seguida, fez-se um estudo comparativo entre os pacientes internados e em tratamento ambulatorial. Avaliou-se a possibilidade de diferenças estatisticamente significantes entre esses dois grupos com respeito às características pessoais e de sensação à dor. Para análise da dor foi utilizado o Questionário de dor McGil, e para as analises estatísticas foram utilizados os testes Qui-quadrado e Exato de Fisher, e os testes T e Mann Whitney. A avaliação do estudo mostrou que, nos dois grupos estudados com relação à dor, não existiu diferença quanto ao sexo, idade e causa da doença renal. Tal população de pacientes, quando internada, apresenta uma percepção mais acurada da dor, e tende a vivenciá-la mais intensamente. O grupo de pacientes em tratamento ambulatorial é constituído por uma população com mais tempo de tratamento em hemodiálise, e tende a apresentar sintomas de dor com maior gravidade.

Palavras chaves: Insuficiência Renal Crônica. Hemodiálise. Dor. McGil. Hospital.

\begin{abstract}
A search on pain in patients with chronic renal failure program hemodiálise.For this study were selected 40 adult patients diagnosed with chronic kidney disease stage V. The selection of patients was done through analysis of data from medical records and laboratory tests. The study used an experimental observational design performed in 20 patients on hemodialysis in the outpatient clinic Cenemge-Belo Horizonte / MG, and 20 patients with chronic renal failure at the Hospital Belo Horizonte, in Belo Horizonte City / MG.The descriptive analyzes of patients initially considered as a single group, "carriers of chronic renal failure on hemodialysis." Then made a comparative study between the inpatient and outpatient treatment. We evaluated the possibility of significant differences between these two groups with respect to the personal characteristics and feeling the pain. For analysis of the pain questionnaire was used McGil of pain, and the statistical analysis we used the chi-square and Fisher's exact, and tests and Mann Whitney. The evaluation study showed that the two groups with regard to pain, there was no difference in sex, age and cause of kidney disease. This population of patients, when admitted, presents a more accurate perception of pain, and tends to experience it more intensely. The group of patients in outpatient treatment consists of a population over time undergoing dialysis treatment, and tends to have pain symptoms with greater severity.
\end{abstract}

Key words: Chronic Renal Failure, Dialysis, Pain, McGil. Hospital. 


\section{INTRODUÇÃO}

Pacientes portadores de insuficiência renal crônica em programa de hemodialise apresentam variadas e recorrentes queixas de dor. Esta população apresenta elevada incidência de doença óssea e perda progressiva de massa muscular, em parte decorrente da característica debilitante própria doença renal crônica (DAUGIRDAS; BLAKE; ING, 2003). Além disto, apresenta também elevada incidência de outras doenças crônicas debilitantes como o diabetes mellitus, as doenças neurológicas, as obstruções vasculares, dentre outras. Estas doenças também contribuem para o aparecimento de dor naqueles pacientes. Além disto, durante as sessões de hemodiálise ocorrem várias intercorrências relacionadas ao tratamento, sendo que as mais comuns são: hipotensão (20-30\%), câimbras (5-20\%), náuseas e vômitos (5-15\%), cefaleia (5\%), dor no peito (2-5 \%), dor lombar (2-5\%), prurido $(5 \%)$,febre e calafrios $(<1 \%)$. (DAUGIRDAS; BLAKE; ING, 2003). As dores perfazem cerca de $35 \%$ do total de queixas apresentadas pelos pacientes durante as sessões de hemodiálise. Uma grande parcela desses sintomas dolorosos tem seu mecanismo fisiopatológico conhecido, podendo ser tratados adequadamente no decorrer do tratamento: a dor muscular que se segue às câimbras, ocasionadas por remoção rápida de líquido corporal; as cefaleias, associadas às elevações da pressão arterial; a dor torácica que ocorre na chamada síndrome da reação ao dialisador, antes conhecida como síndrome de primeiro uso. Tal situação, em particular, ocorre quando se inicia um novo tratamento dialítico e ocorre uma resposta inflamatória sistêmica. (DAUGIRDAS; BLAKE; ING, 2003). De grande importância é o quadro de dores provocado pelas alterações ósseas patológicas que ocorrem nos portadores de insuficiência renal crônica. Essas alterações ósseas são agrupadas sob o termo Osteodistrofia Renal, que engloba as desordens esqueléticas secundárias às alterações do metabolismo de cálcio e fósforo, e as consequentes remodelações ósseas que se seguem (MATSUO et al.,1985). As alterações ósseas podem provocar dores difusas, progressivas, com acometimento de coluna vertebral, joelhos, tornozelos e coxas, podendo levar à imobilidade total. Também como causa de dores ósseas e musculares temos a síndrome musculoesquelética, que se manifesta em pacientes que se encontram por longos anos em hemodiálise, e que seria provocada pela deposição de amiloide e beta 2-microglobulina. (MATSUO et al.,1985) Portanto, a dor neste contexto tem múltiplas causas e manifestações variadas. A presente pesquisa investigou a manifestação de dor no portador de insuficiência renal crônica em hemodiálise, procurando evidenciar os seus vários desdobramentos no quadro clínico da doença renal crônica. 


\section{MÉTODO}

Foi utilizado um desenho experimental observacional, realizado em 20 pacientes em programa de hemodiálise ambulatorial, vinculados à Clínica Cenemge-Belo Horizonte/MG, e em 20 pacientes portadores de insuficiência renal crônica internados no Hospital Belo Horizonte-Belo Horizonte/MG, em hemodiálise intra-hospitalar. Selecionados 40 pacientes adultos, com diagnóstico de insuficiência renal crônica, definida como a perda irreversível da função renal, por um período superior a 3 meses, e com um clearence de creatinina $<15 \mathrm{~mL} /$ minuto. Os pacientes ou estavam em programa de hemodiálise ambulatorial na Clínica Cenemge, ou internados e em programa de hemodiálise intra-hospitalar no Hospital Belo Horizonte. Os mesmos concordaram em participar assinando o Termo de Consentimento Livre e Esclarecido (TCLE) específico para este trabalho. Foram excluídos pacientes com fraturas recentes ou em consolidação, os portadores de doenças psiquiátricas, pacientes com doenças neoplásicas ou em condições clínicas graves, os menores de idade e os analfabetos. Também foram excluídos os pacientes portadores de insuficiência renal aguda.

\section{RESULTADOS E DISCUSSÃO}

Foram estudados 40 pacientes, sendo, 19 mulheres $(47,5 \%)$ e 21 homens $(52,5 \%)$. A idade variou de 21 a 80 anos, com idade média de 57 anos. Com relação à cor da pele, houve um predomínio de não-brancos no grupo em tratamento ambulatorial, e uma maior concentração de brancos entre os pacientes internados. A escolha da amostra de pacientes foi feita através de análise de dados de prontuário e de exames laboratoriais. Foi aplicado um desenho de questionário, e estes dois grupos foram comparados com respeito às variáveis de perfil e à sensação de dor, sendo utilizado como instrumento de avaliação o Questionário de Dor McGill. Para realizar estas comparações foram utilizados os testes Qui-quadrado e Exato de Fisher para as variáveis não-numéricas e os testes "T" e Mann Whitney para as variáveis numéricas. Foram comparadas as variáveis de perfil (sexo, cor, idade, etc.) e, posteriormente, o número de descritores e o índice de dor. A análise da amostra de pacientes com insuficiência renal crônica foi dividida em dois grupos, sendo um grupo de pacientes em tratamento ambulatorial, e o outro grupo de pacientes internados no hospital. Foi realizado um estudo comparativo entre os pacientes internados e os que estavam em tratamento ambulatorial, e verificou se havia diferenças estatisticamente significantes entre esses dois grupos.

\subsection{Sexo}

A distribuição da população estudada, quando definida por sexo, mostrou um equilíbrio entre homens e mulheres, com um 
ligeiro predomínio de homens, sem diferença estatisticamente significativa. $O$ percentual de mulheres para o grupo em tratamento ambulatorial foi um pouco maior que para o grupo internado, 52,5\% contra 47,5\%. Porém, não existiu diferença estatisticamente significativa. Este é um resultado relativamente esperado, pois o gênero não deve influenciar a chance.

Figura 1 - Distribuição dos pacientes em relação ao sexo.

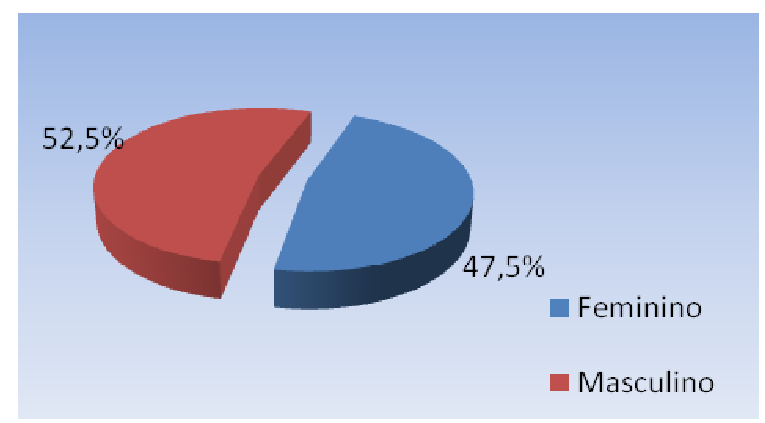

Fonte: Os autores.

Figura 2 - Análise das respostas do índice da dor quanto ao gênero.

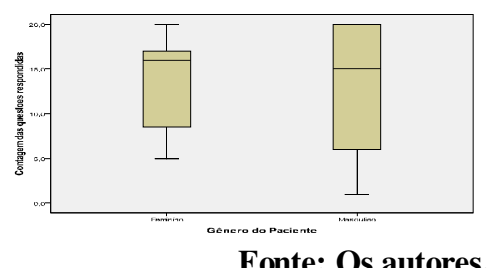

\subsection{Idade}

A idade média do grupo avaliado foi de 57 anos, com 50\% dos pacientes com 60 anos ou mais. O paciente mais jovem tinha 21 anos e o mais velho 80 anos. Não houve diferença estatisticamente significativa comparando-se o grupo de pacientes em tratamento ambulatorial e o grupo de pacientes internados em hemodiálise.
Figura 3 - Análise comparativa dos pacientes com relação à idade.

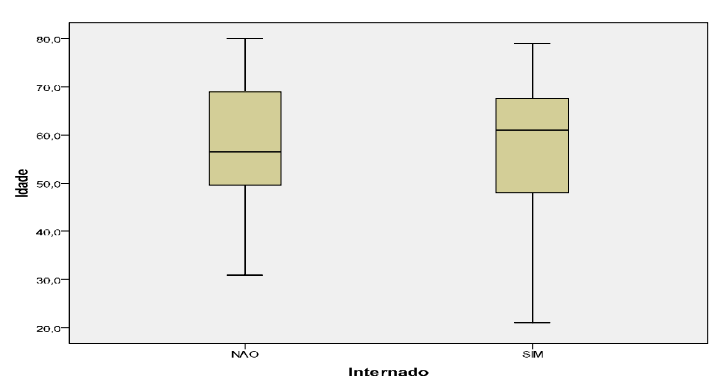

Fonte: Os autores.

Figura 4 - Análise das respostas do índice da dor quanto à idade dos pacientes.

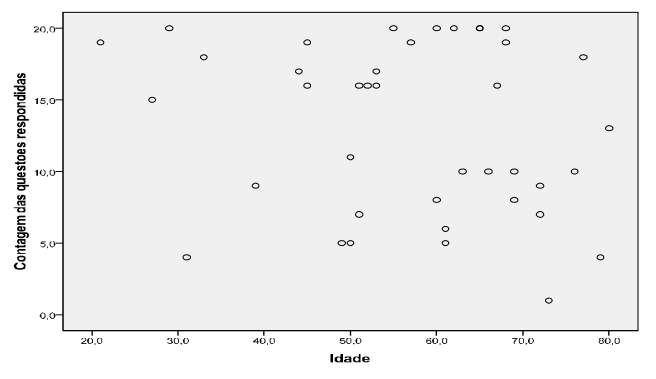

Fonte: Os autores.

\subsection{Cor}

Para a variável cor, houve uma diferença entre os dois grupos avaliados. Existiu uma diferença estatisticamente significativa entre os dois grupos no percentual de negros, onde o grupo em tratamento ambulatorial apresentou maior concentração de negros do que o grupo internado. $\mathrm{O}$ grupo em tratamento ambulatorial mostrou $30 \%$, de negros contra $0 \%$ no grupo de pacientes internados. Verificando-se as diferenças entre raças com respeito ao número de descritores e ao índice geral de dor, observa-se que não houve diferenças com respeito à média de número de descritores e ou para o índice médio de dor. De acordo com 
Weisbord (2007), apesar de alguns estudos terem mostrado que pacientes negros em hemodiálise tivessem uma melhor qualidade de vida global do que os brancos, as diferenças raciais na carga de sintomas não estão bem caracterizados. Mark Nruh (2004) não encontrou diferença estatisticamente significativa entre negros e não negros com relação aos sintomas em hemodiálise. Conforme Price (1997) existem vários parâmetros relativos à hemodiálise que diferenciam de forma estatisticamente significativa negros e não-negros, notadamente qualidade de vida global e incidência de morte, maiores nos negros. Somnath et al. (2007) demonstraram que caucasianos tendem a apresentar dor mais severa que os afroamericanos. Nesse trabalho os achados nos diz que não foi evidenciado diferenças estatisticamente significativas em respeito à manifestação de dor entre negros e não negros.

\section{Figura 5 - Distribuição dos pacientes com relação à} cor.

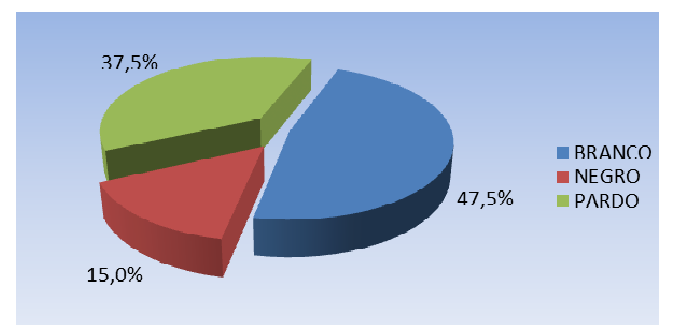

Fonte: Os autores.

\subsection{Tempo de Hemodiálise}

O grupo em tratamento ambulatorial tem média mais elevada para o tempo de hemodiálise que o grupo internado. A diferença entre os grupos com respeito ao tempo de hemodiálise é estatisticamente

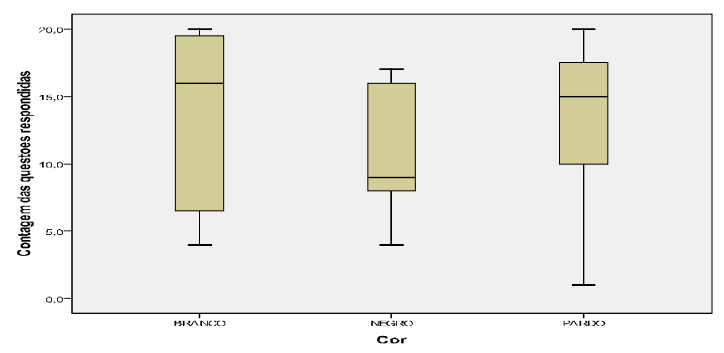

Figura 6 - Análise das respostas do índice da dor quanto à cor entre pacientes internados e não internados.

Fonte: Os autores.

significativa. O grupo avaliado tem 15 pessoas com até 01 ano e 25 com mais de 01 ano de hemodiálise. As medidas descritivas foram muito mais elevadas para este grupo. A maior incidência de dor no grupo de pacientes com mais tempo em hemodiálise corrobora o que já é encontrado na literatura. Esta população tende a apresentar doenças ósseas mais graves, e uma maior incidência de hiperparatireoidismo (SOMNATH et al., 2007). De acordo com Schwarz C (2006), o hiperparatireoidismo leva ao aparecimento de osteodistrofia, calcifilaxia e deformidades esqueléticas, e tem relação direta com o tempo de existência da doença renal. Por esta razão, e subsidiado pelos dados de literatura, foi encontrado uma maior manifestação de dor nos pacientes ambulatoriais.

Figura 7 - Análise das respostas do índice da dor quanto à cor entre pacientes internados e não internados.

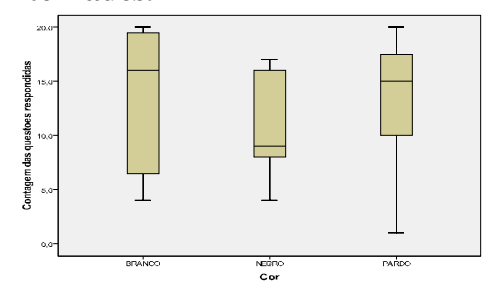

Fonte: Os autores. 


\subsection{Causas da doença renal}

Os dois grupos têm percentuais muito semelhantes para as causas da doença renal. As causas apontadas são semelhantes nos dois grupos, com um predomínio de glomerulonefrite crônica, seguida de Diabetes Mellitus e hipertensão arterial. Não houve diferenças estatisticamente significativas entre os grupos com relação à dor. Verificando-se as diferenças entre as causas da doença renal em relação ao índice geral de dor, observa-se que o número de descritores e o índice de dor não dependem da causa da doença relatada pelo paciente. Davinson SN (2003) relata que os doentes negros e os não-negros apresentam grande diversidade de doenças causadoras de insuficiência renal crônica, algumas mais prevalentes em negros, como a hipertensão arterial, e outras mais prevalentes em brancos, como o diabetes mellitus. No entanto, observando-se as causas da doença renal, não houve diferença estatisticamente significativa com relação à dor.

Figura 8 - Análise do número de descritores e do índice da dor com a causa da doença renal.

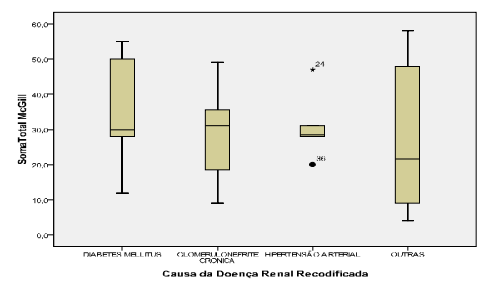

Fonte: Os autores.

\section{CONCLUSÕES}

A análise comparativa entre pacientes em hemodiálise ambulatorial e pacientes em hemodiálise intra-hospitalar mostrou que existem algumas diferenças estatisticamente significativas entre os dois grupos investigados, no que se refere à cor, ao tempo de duração da doença renal e à percepção da dor, mais nítida no grupo de pacientes ambulatoriais. As diferenças também ocorreram com relação ao tempo de duração da doença, sendo mais elevada nos pacientes em tratamento ambulatorial. Também apresentaram diferenças estatisticamente significativas as descrições de dor, mais detalhadas no grupo de pacientes internados e mais severas no grupo de pacientes em tratamento ambulatorial. $\mathrm{Na}$ avaliação da população de pacientes portadores de insuficiência renal crônica, com relação à dor, não existe diferença quanto ao sexo, idade $\mathrm{e}$ causa da doença renal. Estes pacientes, quando internados, tendem a ter uma percepção mais acurada da dor, e, em consequência, vivenciála mais intensamente. $\mathrm{O}$ grupo de pacientes em tratamento ambulatorial é representado por uma população com mais tempo de doença renal e apresenta sintomas de dor com maior gravidade. A presente pesquisa aponta para a necessidade de implementação de tratamentos mais eficazes para o alívio da dor desses pacientes, uma vez que a persistência de sintomas dolorosos leva à utilização de medicamentos e equipamentos, além da 
mobilização de profissionais, com elevados custos financeiros para todo o sistema de saúde e para o paciente. A despeito da melhora das tecnologias em diálise, da incorporação de novas técnicas e dos novos conhecimentos, a dor dos pacientes em hemodiálise permanece comum e frequente. Portanto, torna-se necessária especial atenção às queixas de dor apresentadas por este grupo de pacientes, com instituição de novos procedimentos para o tratamento desta manifestação clínica.

\section{REFERÊNCIAS}

BARDIN T. Rheumatologic complications and amyloidosis in dialysis patients. Ver. Praticien 40:619-624,1990.

BENNETT MI. Theories, history and current taxonomy.In: Bennett MI (ed). Neuropathic pain. Oxford University Press, p. 3-9, 2006.

BINDI P; CHANARD J. Destructive spondyloarthropathy in dialysis patients: an overview. Nephron 55:104-109, 1990.

BRASIL. Ministério da Saúde. Estudo epidemiológico brasileiro sobre terapia renal substitutiva. Ministério da Saúde, Secretaria de Assistência à Saúde-Versão preliminar. Brasília, 2006.

BRUCKNER FE; BURKE M;PEREIRA RS; BENDING M;, WATSON B. Synovial amyloid in chronic hemodialysis contains beta-2-microglobulin. Ann Rheum Dis 46:634-637, 1987.

BUSH WH JR, CHOYKE PL, BLUTH RI, CASALINO DD, FRANCIS IR, JAFRI SZ, KAWASHIMA A, KRONTHAL A, OLDER RA, PAPAINCOLAOU N, RAMCHANDANI $\mathrm{P}$, ROSENFIELD AT, SANDLER CM, SEGAL AJ, TEMPANY C, RESNICK MI. Expert Panel on Urologic Imaging. Renal failure. Reston (VA): American College of
Radiology (ACR); 2005. 8p. In: Current Guideline Summary: ACR Appropriateness Criteria ${ }^{\circledR}$ renal failure. NGC:007019

CAMPISTOL JM; SOLE M; MUNOZGOMEZ J; LOPEZ-PEDRET REVERT $\mathrm{L}:$ Systemic involvement of dialysis amyloidosis. Am. J. Nephrol 10:389-396, 1990.

CANAUD B;BOSC JY;LERAY H; MORENA M;, STEC FM. Dialysate : rationale and technical aspects. In: Chronic Inflammatory Disease in Hemodialysis. S Karger AG, Switzerland, 2000.

DANESH F; HO LT. Dialysis-related amyloidosis: history and clinical manifestations Semin Dial. Mar-Apr;14(2):805, 2001.

DANESH F;KLINKMANN J;YOKOO H; IVANOVICH P. Fatal cervical spondyloarthropathy in a hemodialysis patient with systemic deposition of $\beta 2$-microglobulin amiloid. Am J Kidney Dis 33:563-566, 1999.

DAURGIDAS JOHN T; BLAKE G. PETER; ING S. TODD. Handbook of Dialysis. 3rd edition, Lippincott Willians \& Wilkins, 2003. DAVISON SN. Chronic kidney disease: psychosocial impact of chronic pain.

Geriatrics. 2007 Feb;62(2):17-23

DI RAIMONDO CR; CASEY TT; DI RAIMONDO CV; STONE WJ . Pathologic fractures associated with idiopathic amyloidosis of bone in chronic hemodialysis patients. Nephron 43:22-27,1986.

DOR. In: Holanda Ferreira, Aurélio Buarque. Novo Aurélio, O Dicionário da Língua Portuguesa. Ed.3 ${ }^{\text {a }}$, Nova Fronteira, 1999.

DOR. In: Houaiss, Antonio. Novo Dicionário Houaiss da Língua Portuguesa, Editora Objetiva, 2009.

DOR. In: Michaelis Língua Portuguesa Dicionário Escolar: Nova Ortografia, $1^{\mathrm{a}}$ edição, Editora Melhoramentos, 2008. 
GAL R; KORZETS A;SCHWARTZ A; RATH-WOLFSON L;GAFTERU. Systemic distribution of $\beta 22$-microglobulin-derived amyloidosis in patients who undergo longterm hemodialysis. Arch Pathol Lab Med 118:718-721,1994.

GEJYO F; YAMADA T; ODANI S; NAKAGAWA Y;, ARAKAWA M; SHIRAHAMA T; COHEN AS; SCHIMID K: A new form of amyloid protein associated with hemodialysis was identified as $\beta 2$ microglobulin. Biochem Biophys Res Commun 129:701-706,1985.

GORDON DA; PROZANSKI W; OGRYZLO MA. Synovial fluid examination for the diagnosis of amyloidosis. Ann Rheum Dis 32:428-430,1973.

HURST N P; VAN DEN BERG R ; DISNEY A; ALCOCK M; ALBERTYN L; GREEN M; INTERNATIONAL ASSOCIATION FOR STUDY OF PAIN. Classification of Chronic Pain. Second Edition. IASP Task Force on taxonomy, edited by H. Merskey and N. Bogduk, IASP Press, Seattle, 1994.

JADOUL M; GARBER C; NOEL H,SENNESAEL J; VAN-HOLDER R; BERNAERT P; VAN YPERSELE DE STRIHOU C. Histological prevalence of $\beta 2$ microglobulin amyloidosis in hemodialysis : a prospective post-mortem study. Kidney Int. 51:1928-1932.1997.

KAVANNAGH TG; DALY KE. Bone cyst in the cervical spine due to secondary amyloidosis. Acta Orthop Scand 63:221-222, 1992.

KAWANO M;MURAMOTO H;YAMADA M; MINAMOTO M; ARAKI ;MABUCHI H; NONOMURA A. Fatal cardiac $\beta 2-2$ microglobulin amyloidosis in patients on longterm hemodialysis. Am.J. Kidney Dis 31:E4,1988.

KENZORA JE. Dialysis carpal tunnel syndrome. Orthopedics 1:195-203, 1978.
KOCH, M Karl. Dialysis-related amyloidosis. Kidney International (1992) 41, 1416-1429; doi: 10.1038/ki; 207, 1992. 104

KUNTZ D;VEAU B; BARDIN T; DRUEKE T; TREVES R ;DRYLL A. Destructive spondylarthropathy in hemodialysed patients. A new syndrome.Arthritis Rheum 27:369375,1984 .

LONNEMANN G; KOCH KM. Beta2microglobulin amyloidosis: effects of ultrapure dialysate and type of dialyzer membrane. J.Am. Soc. Nephrol. jan;13 Suppl 1:S72-7, 2002.

MARK U NRUH , D ANA M ISKULIN , G UOFEN Y AN , R ON D H AYS , R OBERT B ENZ , J OHN W K USEK AND K LEMENS B M EYER. The hemo study g roup, racial differences in health-related quality of life among hemodialysis patients. Kidney International 65, 1482-1491, 2004.

MATSUO K, NAKAMOTO M, YASUNAGA C, GOYA T, SUGIMACHI K. Dialysisrelated amyloidosis of the tongue in long-term hemodialysis patients. Kidney Int 52:83241,1997. Complication. Ann Rheum Dis. 44:729-733, 1985.

MELZACK R. The McGill pain questionnaire: major properties and scorin methods, Pain, v.1. n.3. p.277-99, 1975.

MELZACK R; KATZ J. Pain assessment in adult patients. In: Wall and Melzack's Textbook of Pain -- McMahon SB; Koltzenburg M; eds. 5th Edn. London: Elsevier. 291-30, 2006.

MENEREY K;BRAUNSTEIN E;,BROWN M;FOX IH. Musculoskeletal symptoms related to arthropathy in patients receiving dialysis. $\mathrm{J}$. Rheumatol. 15:1848-1854,1988.

MUNOZ-GOMEZ J;BERGADA-BARADO E; GOMEZ-PEREZ R; LLOPART-BUISAN E ; SOLE-ARQUES M. Amyloid arthropathy in patients undergoing periodical aemodialysis for chronic renal failure: a new complication. Ann Rheum Dis. 44:729-733,1985. 
MYATA T; INAGI R; IIDA Y; SATO M; YAMADA N; ODA O; MAEDA K;SEO H. Involvement of beta-2-microglobulin modified with advanced glycation end produts in the pathogenesis of hemodialysis-associated amyloidosis.Induction of human monocyte chemotaxis and macrophage secretion of tumor necrosis factor-alfa and interleukin-1. J Clin Invest ; 93:521-528, 1994.

NATIONAL KIDNEY FOUNDATION. KDOQI. Clinical Practice Guidelines for Chronic Kidney Disease: Evaluation, Classification, and Stratification, Inc, 2002.

NEMANJA JONJIC, VIDOJKO DJORDJEVIC, NEBOJSA DJORDJEVIC AND STANIMIR LJUBENOVIC. Effect of hemodialysis duration, age and gender on emergence of renal osteodystrophy symptoms. Acta Medica Medianae 2008,vol.47:19-24. 105

NOEL LH; ZINGRAFF J,BARDIN T; ATIENZA C, KUNTZ D; DRUEKE T. Tissue distribution of dialysis amiloidosis. Clin Nephrol 27:175-178,1987.

PIMENTA, C. A. M.; TEIXEIRA, M. J. Questionário de dor McGill: proposta de adaptação para a língua portuguesa.

Rev.Esc.Enf.USP, v.30. n.3, p. 473-83, dez. 1996.

PRICE DA, OWEN WF JR. AfricanAmericans on maintenance dialysis: A review of racial differences in incidence, treatment, and survival. Adv Ren Replace Ther. 1997 Jan. 4(1):3-12.

RINSHO BYORI. Beta 2-microglobulin and dialysis-related amyloidosis. The Japanese journal of clinical pathology. vol.49(3):244248, 2001.

ROMÃO JUNIOR, João Egidio. Doença Renal Crônica: Definição, Epidemiologia e Classificação. J Bras Nefrol vol.XXVI - n 3 - Supl. 1 - ago. 2004.

ROUSSELIN B; HELENON O; ZINGRAFF J;DELONS S; DRUEKE T; BARDIN T;
MOREAU JF. Pseudotumor of the craniocervical junction during long-term hemodialysis. Arthrithis Rheum33:1567$1573,1990$.

SARA N; DAVISON MD. Pain in hemodialysis patients: prevalence, cause, severity, and management. American Journal of Kidney Diseases vol.42(6):1239-1247, dez. 2003.

SETHI D; HUTCHISON AJ; CARY NR; BROWN EA; CURTIS JR; WOODROW DF. Macroglossia and amyloidoma of the buttock: evidence of systemic involvement in dialysis amyloid. Nephron. 55:312-315, 1990.

SOCIEDADE BRASILEIRA DE NEFROLOGIA. Censo Brasileiro de Diálise, 2008.

SCHWARZ C; SULZBACHER I; OBERBAUER R. Diagnosis of renal osteodystrophy. European Journal of Clinical Investigation. Vol.36(s2):13-22, aug. 2006.

SOMNATH SAHA; MICHELE FREEMAN; JOAHD TOURE; KIMBERLY M. TIPPENS; CHRISTINE WEEKS. Racial and Ethnic Disparities in the VA Healthcare System:A Systematic Review. Health Services Research \& Development Service, jun. 2007. 106

STRIHOU, C Y; JADOUL, M; MALGUEM, $\mathrm{J}$; MALDAGUE, B; JAMART, J. Effect of dialysis membrane and patient's age on signs of dialysis-related amyloidosis. The working party on dialysis amyloidosis; KIDNEY INT. May;39(5):1012-9,1991.

ULLIAN ME; HAMMOND WS; ALFREY AC; SCHULTZ A; MOLITORIS BA. $\beta 2$ microglobulin-associated amyloidosis in chronic hemodialysis patients whit carpal tunnel syndrome. Medicine. 68:107-115,1989.

VANYPERSELE DE STRIHOU C; HONHON B; VANDENBROUCKE JM; HUAUX JP; MALDAGUE B. Dialysis amyloidosis. Adv Nephrol Necker Hosp 17:401-421,1988. 
VINCENT C;REVILLARD JP;GALLAND

M. Serum $\beta 2$-microglobulin in hemodialysed patients. Nephron 10:320-331,1973.

WARREN DJ; OTIENO LS. Carpal tunnel syndrome in patients on intermittent hemodialysis. Postgrad Med J 51:450-452, 1975.

ZHOU H; PFEIFER U; LINKE R. Generalized amyloidosis from $\beta 2$ microglobulin, with caecal perfuration after long-term hemodialysis. Virchows Arch A Pathol Anat Histol 419:349-353,1991. 